\title{
THE INFLUENCE OF MOTOR PREPARATION ON THE RESPONSE OF CEREBELLAR NEURONS TO LIMB DISPLACEMENTS ${ }^{1}$
}

\author{
PETER L. STRICK
}

Research Service, Veterans Administration Medical Cenler, and Departments of Neurosurgery and Physiology, State University of New York-Upstate Medical Center, Syracuse, New York 13210

Received January 22, 1982; Revised April 29, 1983; Accepted April 29, 1983

\begin{abstract}
This study examined the influence of "motor set" on the response of neurons in the deep cerebellar nuclei to peripheral afferent inputs. Two monkeys were trained to perform arm movements which were triggered by imposed displacements of their forelimb. Imposed displacements were generated by a torque motor which was coupled to a handle held by the monkey. The direction of the triggered movements depended on a prior instruction presented to the animal before the onset of the imposed displacement.

Single neuron recordings were made from 105 interpositus and 191 dentate neurons whose activity was related to the performance of the task. Forty-three of the interpositus and 46 of the dentate neurons displayed a change in activity within $70 \mathrm{msec}$ of the onset of the imposed displacement. None of these neurons displayed a neural response following the onset of the instruction.

The short latency response of most interpositus neurons was related to the parameters of the afferent input generated by the imposed displacements. For more than $60 \%$ of the interpositus neurons this response was strongly influenced by the direction of the imposed displacement. None of these responses were markedly altered by the prior instructions.

In contrast, the short latency response of $67 \%$ of the dentate neurons, although triggered by the imposed displacement, was markedly altered by the "motor set" of the animal. For most of the dentate neurons whose activity was influenced by the animal's "motor set," the neural response was contingent on two factors: (1) the prior instruction given to the animal and (2) the direction of the imposed displacement. In a second set of dentate neurons (13\% of those influenced by motor preparation) the characteristics of the short latency response were determined solely by the nature of the prior instruction. For these neurons the imposed displacement served as a trigger for possible changes in the activity. Thus, dentate neuron activity can behave like a motor command signal which is triggered at short latency by a peripheral event but depends on the intent of the animal.
\end{abstract}

A major goal of motor systems physiology is to better understand how peripheral afferent inputs and central command signals interact to initiate and control motor behavior. In some situations afferent input is utilized largely as a feedback signal to regulate movement. In these instances motor output is, in part, a replica of the afferent input. For example, movements regulated on

\footnotetext{
${ }^{1}$ 'The cerebellar recordings were performed while I was a Staff Fellow in the Laboratory of Neurophysiology, National Institute of Mental Health. I thank the members of this lahoratory and particularly Dr. Edward V. Evarts for their valuable assistance. I also thank Drs. James B. Preston and Donna S. Hoffman for their critical review of this manuscript and Ms. Cathryn Skretch for her assistance in the preparation of the figures. The data analysis and preparation of this manuscript were supported by the Medical Research Service of the Veterans Administration.
}

this basis show "local sign;" i.e., the direction of the afferent input specifies the direction of the resultant motor response. In other situations, afferent input functions to "trigger" volitional responses. In these cases the resultant movements are determined largely by the prior motor preparation or "set" of the animal (cf. Creed et al., 1932; Hammond, 1956, 1960; Fvarts et al., 1971; Crago et al., 1976; Evarts and Granit, 1976; Marsden et al., 1977, 1978; Evarts and Vaughn, 1978; Houk, 1978). At present we know little about the neural basis of either triggered volitional responses or motor preparation.

The interaction between afferent input and central commands occurs at many levels in the central nervous system. Evarts and Tanji (1976) examined the interactions which occur between these signals in the motor cortex. In their study, primates were trained to perform arm movements triggered by imposed displacements of 
the forelimb. The direction of the triggered movement depended on a prior instruction presented to the animal before the imposed displacement. The imposed displacements evoked two types of short latency responses from pyramidal tract neurons (PTNs) in the motor cortex. The first response, which occurred at latencies as short as $20 \mathrm{msec}$, was dependent on the direction of the imposed displacement. Because this response was not influenced by the prior instruction and depended on the pattern of afferent input generated by the imposed displacement, it was termed a "reflex PTN discharge." In contrast, the second response, which occurred at longer latencies (40 to $50 \mathrm{msec}$ ), was not dependent on the parameters of the afferent input which triggered it. Rather, this response depended on the nature of the movement, which the animal performed and was termed an "intended PTN discharge."

Based on the differences between the two responses, Evarts and Tanji (1976) argued that separate central pathways provide the main input for each response. Since the "reflex" discharge was tightly coupled to afferent input, it appeared to be comparable to the responses others have evoked from motor cortex neurons by stimulating cutaneous, muscle, and joint afferents (e.g., Rosen and Asanuma, 1972; Lemon and Porter, 1976; Wong et al., 1978; Fetz et al., 1980; Strick and Preston, 1982). Less is known about the origin of the "intended" P'IN discharge, which was viewed as a manifestation of a central program (Evarts and Tanji, 1976). Evidence from a number of physiological studies suggests that the cerebello-thalamocortical pathway might provide an input for this response (e.g., Meyer-Lohmann et al., 1975; Strick, 1976a, b; Vilis et al., 1976; Thach, 1978).

In the experiments reported here, the interaction between afferent input and central motor programs was examined in two of the deep cerebellar nuclei. Single neurons were recorded in dentate and interpositus while monkeys performed the same task as that cmployed in the motor cortex experiments of Evarts and Tanji, (1976). Both dentate and interpositus neurons responded at relatively short latencies following imposed displacements of the animal's limb. The short latency response of most interpositus neurons was related to parameters of the afferent input generated by the imposed displacements. In contrast, the short latency response of most dentate neurons, although triggered by the imposed displacement, was influenced and, in some cases, determined by the motor "set" of the animal. Thus, the response properties of dentate neurons are consistent with their providing an input for "intended" responses in the motor cortex.

Preliminary reports of some of these results have been presented elsewhere (Strick, 1976c, 1978).

\section{Materials and Methods}

Behavioral procedures. Two rhesus monkeys (Macaca mulatta) were trained on the instruction paradigm first employed by Evarts and Tanji (1974). Aspects of this task are illustrated in Figure 1. Each monkey was seated in a primate chair. The animals were trained to grasp the handle of a manipulandum and push or pull it (using elbow and shoulder extension or flexion) according to task instructions. The output of a precision potentiom- eter which was coupled to the rotating shaft of the manipulandum signaled the position of the animal's limb.

In order to initiate each trial the animal positioned the handle in a central "hold" zone which was electronically determined. The central hold zone corresponded to a 1.0 $\mathrm{cm}$ excursion of the handle. A small white lamp was illuminated when the handle was correctly positioned. After a period of holding which varied unpredictably between 2 and 5 sec, a small red or green lamp was turned on. The red lamp signaled the animal to prepare to push and the green lamp to prepare to pull. Push and pull instructions were varied according to a predetermined pseudo-random order so that the monkey could not anticipate the instruction. The monkey was to remain in the hold zone and perform the movement indicated by the instruction only when it felt the handle move.

A brushless DC torque motor was coupled to the axis of the manipulandum. The torque motor could impose load changes which moved the handle of the manipulandum toward or away from the monkey. These displacements of the handle were the signal for the monkey to perform the movements specified by the prior instruction lamps. If, after the onset of the imposed displacement, the monkey moved the handle through the correct "reward" zone, then the animal was given a fruit juice reward. Thus, the instruction lamps told the animal where to move, whereas the imposed displacements told the animal when to move. Each reward zone was bounded by a mechanical stop and was separated from the central hold zone by $7.5 \mathrm{~cm}$. Additional descriptions of the torque motor and training procedures have been presented previously by Tanji and Evarts (1976).

Recording procedures. When an animal became proficient in the task, it was prepared for chronic single-unit recording using techniques previously described (Evarts, 1968). Briefly, the animal was anesthetized and steel bolts were attached to the skull for restraining the head in the primate chair. A hole was cut in the skull over the dentate and interpositus nuclei. The dura was left intact. A cylinder, $20 \mathrm{~mm}$ in diameter, was attached to the skull to permit chronic single-unit recording. Initially, penetrations with glass-insulated platinum-iridium microelectrodes (Wolbarsht et al., 1960) were made at 1-mm separations in order to locate the two deep cerebellar nuclei. Later penetrations were made at $0.5-\mathrm{mm}$ intervals.

During the recording sessions the animal was allowed to perform the task while the electrode was advanced in the search for task-related neuron activity. When a unit was isolated, its firing pattern was qualitatively analyzed on-line by generating a stimulus-triggered dot raster display on a storage oscilloscope. The activity of every isolated unit was observed for 5 to 10 trials during the performance of the four possible combinations of prior instruction and imposed displacement. If the raster display indicated that the unit had any consistent relation to the task, then unit activity, potentiometer output, and an analogue code indicating the timing and type of various task-related events were recorded on a seven-channel tape recorder. At least 25 trials of each condition were recorded when possible.

Electromyographic (EMG) recordings were made from 

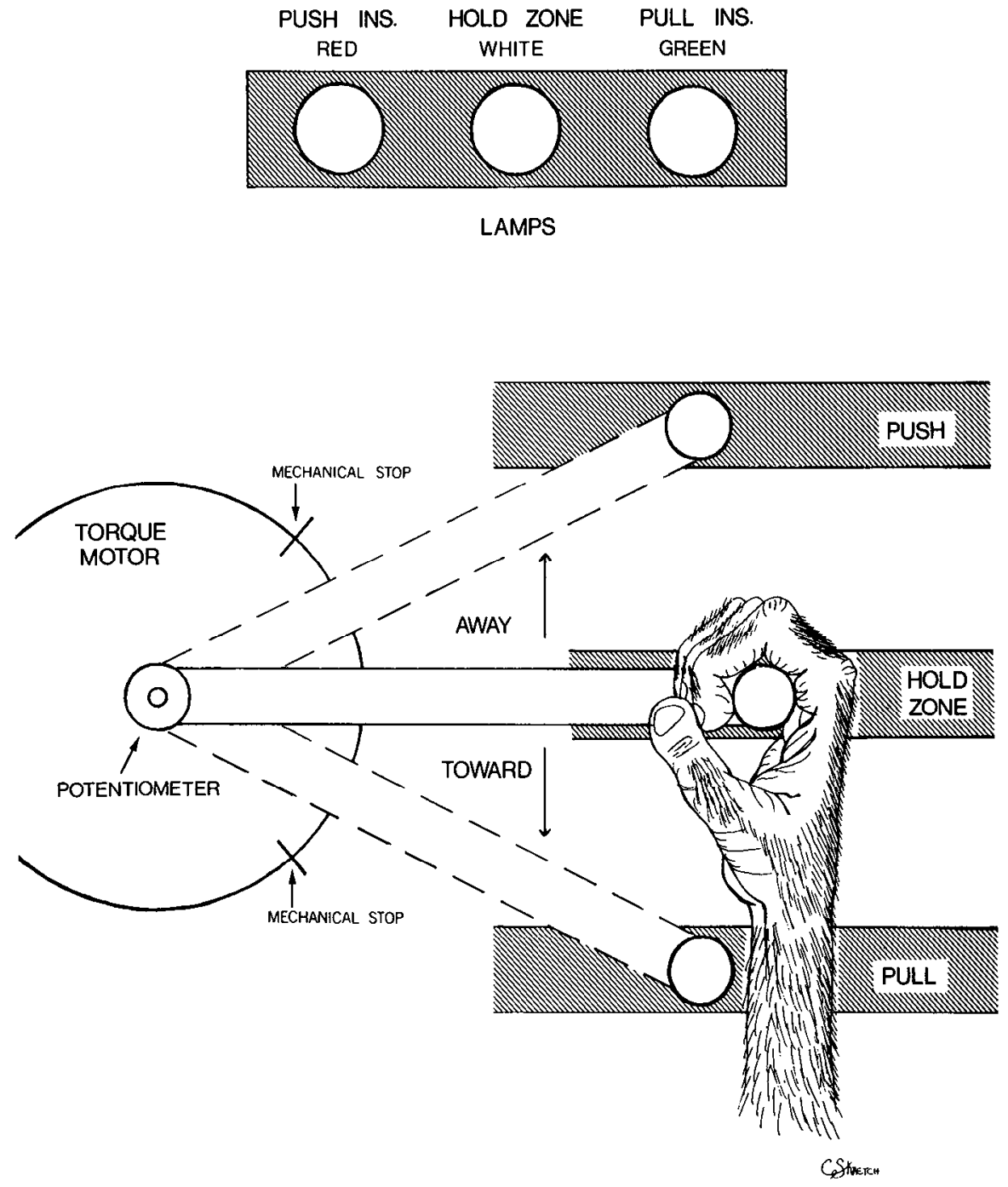

Figure 1. Instruction paradigm. The monkey grasped the handle of a manipulandum which was coupled by a 6 -inch rod to a torque motor. The position of the handle was signaled by a potentiometer coupled to the shaft of the torque motor. The output of the potentiometer was fed to a series of comparators which electronically defined three zones: a central "hold" zone, a "push" reward zone, and a "pull" reward zone. Three lamps were positioned in front of the animal. The white lamp was illuminated when the animal held the handle in the hold zone. When the animal had held in the hold zone for the correct period of time one of the two instruction lamps was illuminated. The red lamp instructed the animal to prepare to push and the green lamp to prepare to pull. Displacements of the handle imposed by the torque motor were the signal for the animal to perform the movement indicated by the prior instruction. See "Materials and Methods" for further details of the task.

muscles of the arm, shoulder, and back during the performance of the task via pairs of teflon-coated wires inserted into individual muscles. For the purposes of analysis, the EMG activity was rectified and fed to a Philbrick-Teledyne voltage-to-frequency converter whose output had a peak frequency of $1000 \mathrm{~Hz}$. This procedure resulted in a frequency-coded representation of muscular activity which could be recorded and analyzed in the same manner as the cerebellar unit data.

Near the conclusion of the experiments, electrolytic lesions were made at selected points in the cerebellar nuclei by passing 10 to $12 \mu \mathrm{A}$ current for 15 to $20 \mathrm{sec}$ through the recording electrode. Animals were later sacrificed with Nembutal, and their brains were perfused with formol/saline. The brains were embedded in cello- idin, cut in the frontal plane at $50 \mu \mathrm{m}$, and then stained with thionin.

Analytical procedures. A PDP-12 computer was used to analyze and display single neuron and EMG activity. The program (developed by W. Vaughn, W. Sherriff, and E. Evarts), together with a description of display, statistical, and analytical procedures, is available from the DECUS Library of the Digital Equipment Corporation. The results of analysis were displayed in the form of rasters and histograms aligned on a number of task events: the onset of the "push" or "pull" instruction and the onset of the two different directions of imposed displacement. Modulations in activity in relation to any of these events were determined with average reciprocal interval (instantaneous frequency) plots of at least 25 
trials. The onset of modulation was determined by a Poisson statistical routine described previously (Tanji and Evarts, 1976). The occurrence of a "neural response" was defined as either an increase or a decrease in spike frequency relative to the period prior to the onset of a task event $(p<0.001)$. When a response was detected, the latency of the response was taken as the time when the $p<0.01$ level was crossed.

\section{Results}

\section{Movement patterns associated with performance of the task}

Figure 2 shows averages of handle position recorded during performance of the task. The four possible combinations of instructions and imposed displacements are illustrated. Although the imposed displacements moved the handle in the same direction in Figure 2, $A$ and $C$, the animal pushed in Figure $2 A$ and pulled in Figure $2 C$ because of the differing prior instructions for the two sets of trials (see also Fig. 2, $B$ and $D$ ). Thus, the direction of the imposed displacement did not specify the direction of the movement it triggered.

Even when prior instructions differed, the trajectories of handle position, following imposed displacements in a particular direction, overlapped for the first $90 \mathrm{msec}$. This observation is illustrated in Figure 3 where averages of handle position for trials triggered by the same direction of imposed displacement have been overlapped. Tonic EMG activity in arm and shoulder muscles prior to a displacement and the first $70 \mathrm{msec}$ of EMG activity evoked by a displacement were unaffected by changes in the prior instruction (see also Tanji and Evarts, 1976; Evarts and Tanji, 1976). These observations suggest that imposed displacements in one direction initially evoke (i.e., for the first $70 \mathrm{msec}$ ) the same somatosensory afferent input, even when prior instructions differ. The present study focused on cerebellar responses occurring in the 70-msec period following the onset of an imposed displacement. This interval will be termed the "analysis period." The influence of the different motor "sets" (created by the two prior instructions) on the response of cerebellar neurons evoked in the analysis period is presented below.

\section{Interpositus and dentate neuron activity}

Recordings were made from 105 interpositus and 191 dentate neurons whose activity changed during the performance of the task. Forty-three of the interpositus and 46 of the dentate neurons displayed a neural response within the analysis period; i.e., within $70 \mathrm{msec}$. of the onset of an imposed displacement, these neurons showed an increase or decrease in activity relative to the 500 msec period prior to the displacement $(p<0.001)$. Each of the 43 interpositus and 46 dentate neurons with a response in the analysis period also was examined for the presence of a neural response following the presentation of the "push" or "pull" instruction. Statistical analysis, as well as visual inspection of rasters and histograms, showed that the onset of an instruction did not evoke a neural response from any of these neurons. In other words, after the onset of an instruction, none of these neurons displayed an increase or a decrease in activity relative to the 1 -sec period prior to the instruction $(p<0.001)$.

Histograms showing the onset times (i.e., when the $p$ $<0.01$ level was crossed) of neural responses triggered by the imposed displacements are presented in Figure 4 . Each neuron was examined during each of the four possible combinations of instructions and imposed dis-
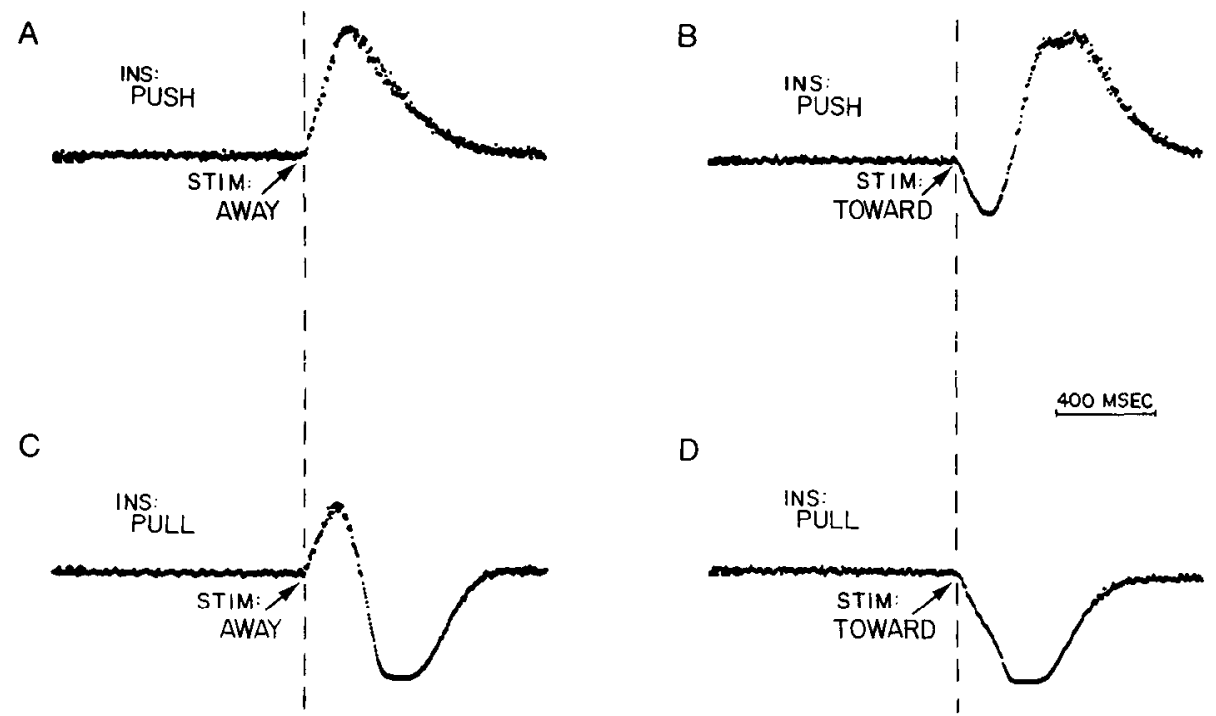

Figure 2. The averages of handle position for the four possible combinations of instruction and imposed displacement. Upward deflections of the trace indicate handle movements away from the monkey (the push direction) and downward deflections indicate movements toward the monkey (the pull direction). The instructions were the same for the trials illustrated in $A$ and $B$ (prepare to push) and for those in $C$ and $D$ (prepare to pull). The imposed displacements were the same for those trials illustrated in $A$ and $C$ (handle moved away from the monkey) and for those in $B$ and $D$ (handle moved toward the monkey). 


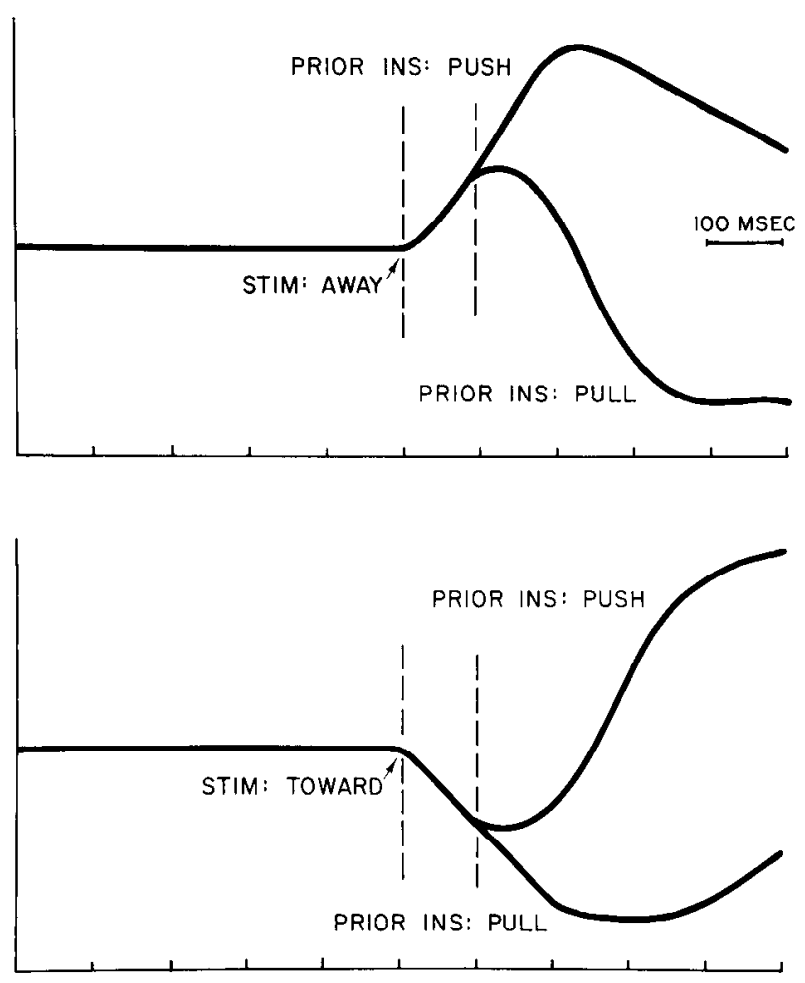

Figure 3. Superimposed averages of handle position. Top, For half the trials the monkey was given the prior instruction to prepare to push and in the other half to prepare to pull. The imposed displacement in both sets of trials moved the handle away from the monkey. Bottom, Same prior instructions as in the top set of trials. The imposed displacement for these trials moved the handle toward the monkey. In both the top and the bottom, the position traces following the same direction of imposed displacement overlap for approximately $90 \mathrm{msec}$ (the period between the dashed lines) even though the prior instructions differed.

placements. Since some neurons did not respond to all four combinations, the total number of responses plotted in the histograms is less than 4 times the total number of recorded neurons. A comparison of the histograms shows that the responses evoked from interpositus neurons tended to occur earlier than those evoked from dentate neurons. The majority of responses of neurons in both nuclei occurred within $50 \mathrm{msec}$ of the imposed displacement. The earliest neural responses occurred 18 msec after the onset of an imposed displacement for an interpositus neuron and $27 \mathrm{msec}$ after the onset of an imposed displacement for a dentate neuron.

Interpositus neuron activity. Each interpositus neuron that displayed a neural response in the analysis period was examined to determine whether this response was altered by prior instructions. The average peak frequency of the neural response evoked by each of the four combinations of imposed displacement and prior instruction during the analysis period was measured from average reciprocal interval (instantaneous frequency) plots. The neural response evoked by a particular direction of imposed displacement when the animal was prepared to push was compared to the response evoked by the same dircction of imposed displacement when the animal was prepared to pull. If the average peak frequencies of the

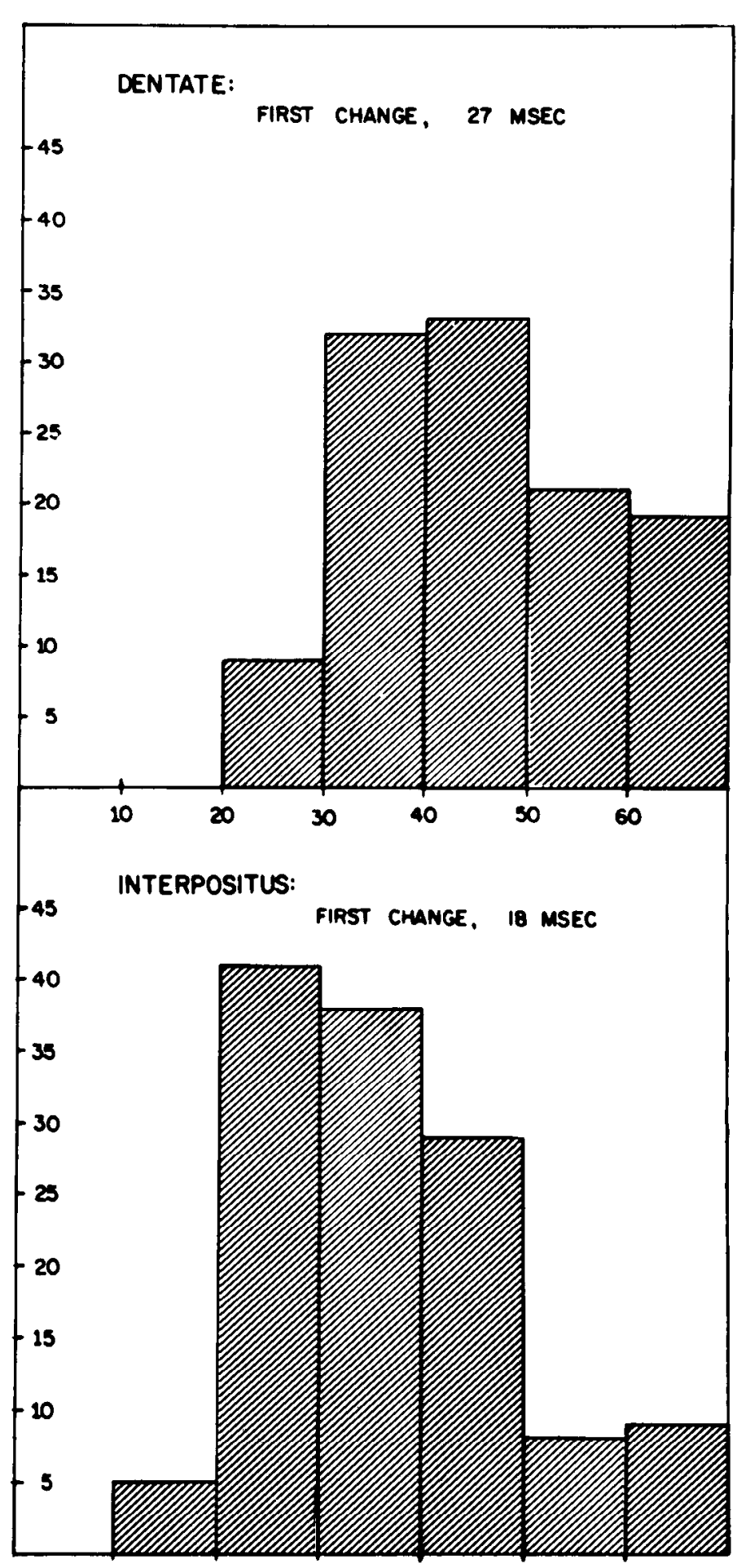

Figure 4. Histograms of the times of change in neural discharge in relation to the onset of the imposed displacement. The ordinate is the time of onset for a neural response (either increase or decrease, $p<0.01$ ) in milliseconds after the onset of the imposed displacement (see "Materials and Methods" for statistical details). The abscissa is the number of changes for dentate (top) or interpositus (bottom) neurons. The number of changes is larger than the total number of neurons since each neuron was observed in all four possible combinations of instruction and imposed displacement. 
responses evoked in the two situations differed by more than $50 \%$, then the neural responses were considered "markedly altered" by prior instructions. Using this criterion, none of the neural responses of interpositus neurons were markedly altered by prior instructions during the analysis period. The largest difference between the average peak frequencies of an interpositus neuron was $16 \%$ and the response of most interpositus neurons ( 39 of 43) differed by less than $10 \%$. Thus, the response of most interpositus neurons in the analysis period showed little or no influence of prior instruction.

Each interpositus neuron that displayed a neural response in the analysis period also was examined to determine whether this response was influenced by the direction of the imposed displacement. If the average peak frequencies of the neural responses evoked by imposed displacements in one direction differed from those evoked by displacements in the opposite direction by more than $80 \%$, then the neural responses were considered "strongly influenced" by the direction of the imposed displacements. Using this criterion, more than $60 \%$ of the interpositus neurons ( 26 of 34 ) with neural responses during the analysis period were strongly influenced by the direction of the imposed displacements. Thus, the pattern of kinesthetic input generated by the imposed displacements determined the short latency response of many interpositus neurons. Eight of the 26 neurons that were strongly influenced displayed neural responses which were reciprocally related to the displacements; i.e., increases in activity were evoked by imposed displacements in one direction and decreases in activity evoked by displacements in the opposite direction.

An example of an interpositus neuron whose responses in the analysis period were reciprocally related to the imposed displacements is illustrated in Figure 5 . The animal was instructed to prepare to push on the trials illustrated in Figure 5, $A$ and $B$, and was instructed to prepare to pull on the trials in Figure $5, C$ and $D$. The imposed displacement moved the handle away from the animal in Figure 5, $A$ and $C$, and toward the animal in Figure $5, B$ and $D$. Independent of the prior instruction, this neuron's activity increased in the analysis period when the imposed displacement moved the handle away from the animal (Fig. 5, $A$ and $C$ ) and decreased in the analysis period when the imposed displacement moved the handle toward the monkey (Fig. $5, B$ and $D$ ). Note that the scales for the individual histograms, in this and other figures, have been varied in order to maximize the illustration of changes in activity. Inspection of this unit's response after the 70 -msec analysis period also revealed neuron activity related to the direction of handle movement. For example, in the trials illustrated in Figure $5 C$, there were three phases of neuron activity which correlated with three phases of handle movement. Neuron activity (1) increased when the handle was moved away from the animal by the imposed displacement, (2) decreased when the monkey, according to the prior instruction, moved the handle toward himself, and (3) increased when the monkey moved the handle away from himself and toward the central hold zone in order to initiate a new trial. Thus, the response of this neuron signaled the change in handle position, irrespective of whether the handle movement was imposed or actively performed. The changes in activity of other interpositus neurons after the analysis period were not examined in detail; however, in most cases such changes were more complicated than those illustrated in Figure 5.

Dentate neuron activity. Using the same criteria as employed with interpositus neurons, each dentate ncuron that displayed a neural response in the analysis period was examined to determine whether this response was altered by prior instructions. The analysis indicated that neural responses for more than two-thirds of the dentate neurons (31 of 46) were markedly influenced by prior instructions. When an animal was prepared to move in different directions, the same direction of imposed displacement could evoke neural responses with substantially different average peak frequencies in the analysis period. For example, the largest difference between the neural responses evoked following the same direction of imposed displacements but opposite instructions, was a change in average peak frequency from 0 to 176 spikes/ sec. The average discharge rate of this neuron when the animal was just holding the handle was 48 spikes/sec. For more than $80 \%$ ( 25 of 31 ) of the dentate neurons which were markedly influenced by prior instructions the average peak frequencies of neural responses differed in the analysis period by more than $75 \%$. Thus, in contrast to interpositus neurons, the neural response of most dentate neurons to kinesthetic input was contingent on the animal's prior motor preparation or "set."

Two types of dentate neurons were observed. For one type ( 4 of 46 neurons), the nature of the prior instruction was the only factor which determined the neuron's response in the analysis period (e.g., Fig. 6). The imposed displacements served only to trigger the short latency responses of these neurons. For the second type of dentate neuron (27 of 46 neurons), both the nature of the prior instruction and the direction of the imposed displacement determined the neuron's response in the analysis period (e.g., Figs. 7 to 9).

An example of the response of the first type of dentate neuron to the four possible combinations of instruction and imposed displacement is illustrated in Figure 6. The change in activity during the analysis period occurred for this unil only when the animal was instructed to prepare to pull. Either direction of imposed displacement triggered a short latency decrease in activity if it followed the pull instruction (Fig. 6, $C$ and $D$ ). When the animal was prepared to push, there was little or no change in this neuron's activity in the analysis period (Fig. 6, $A$ and $B$ ). This was true regardless of the direction of the imposed displacement.

Examples of the second type of dentate neuron whose response was dependent on both the direction of the prior instruction and the direction of the imposed displacement are illustrated in Figures 7 to 9 . For almost two-thirds (20 of 31 ) of these neurons a short latency response was evoked only following one direction of imposed displacement (Figs. 7 and 8). However, the response was dependent on the direction of the prior instruction. For example, the decrease in activity observed during the analysis period for the unit illustrated in Figure 7 occurred only when the animal was instructed to prepare to push and the imposed displacement moved the handle toward the monkey (see Fig. 7A). There was 


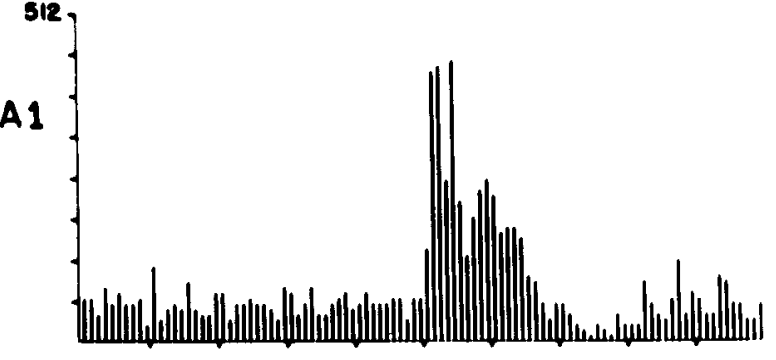

A2
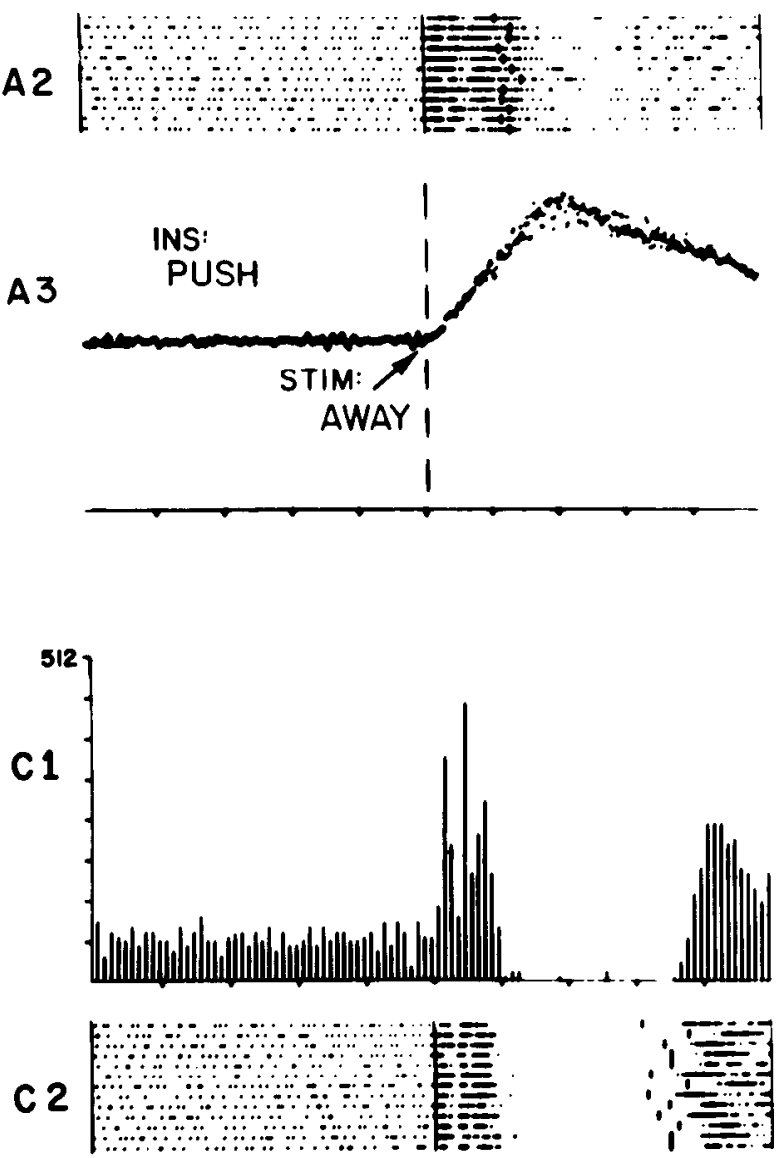

C 2
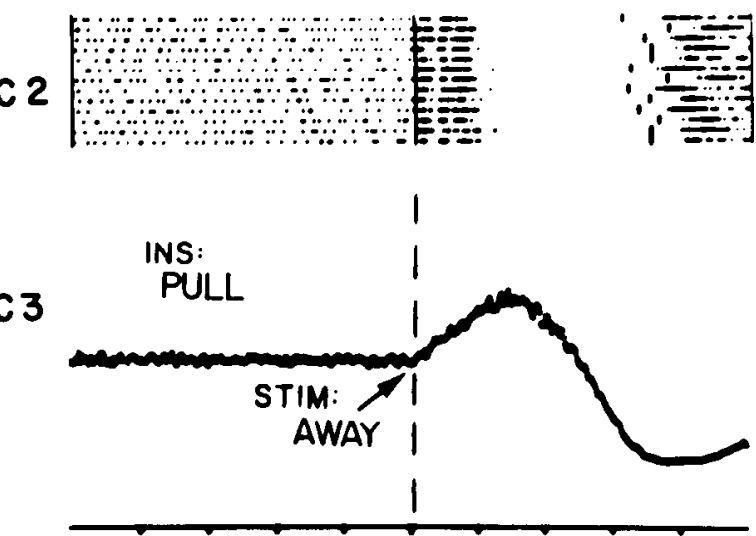
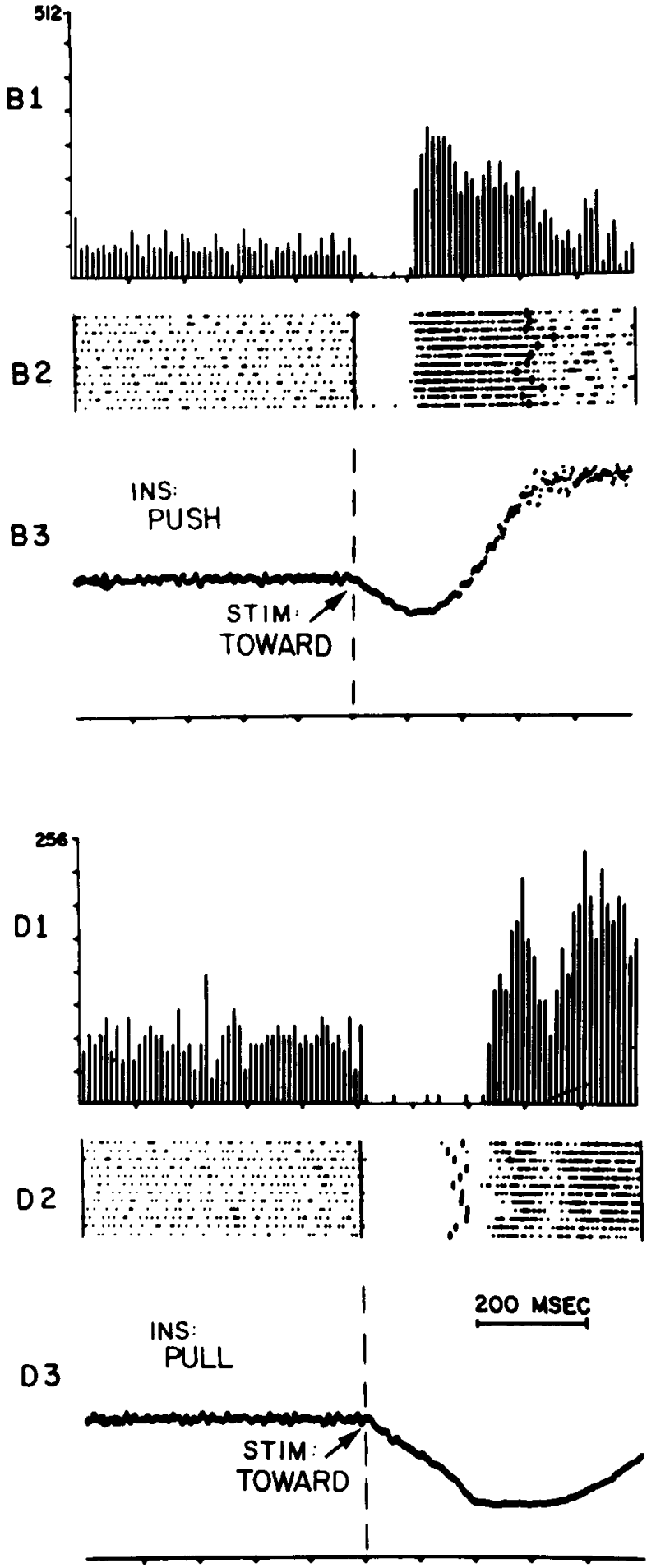

Figure 5. Responses of an interpositus neuron to imposed displacements: Lack of modification by motor preparation. Neural response averages $(A 1, B 1, C 1$, and $D 1)$, rasters of individual trials $(A 2, B 2, C 2$, and $D 2)$, and averages of handle position $(A 3$, $B 3, C 3$, and D3) are shown for recordings from the same interpositus neuron recorded under the four possible combinations of instruction and imposed displacement. In $A$ and $B$, the animal was instructed to prepare to push and in $C$ and $D$, to prepare to pull. In $A$ and $C$, the imposed displacement moved the handle away from the monkey and in $B$ and $C$, the handle was moved toward the monkey. Each line in the rasters represents an individual trial and each dot represents a single unit discharge. The maximum of the scale in $D 1$ (256) differs from that of the other averages (512). Note that this neuron's activity increased when the handle was moved away from the monkey and decreased when the handle was moved toward the monkey. This change in neural activity was independent of the nature of the prior instruction.

little or no change in the neuron's activity in the analysis period when the same imposed displacement was preceded by the "pull" prior instruction. Imposed displacements which moved the handle away from the monkey did not influence this neuron's activity during the analysis period (not illustrated).

A similar pattern of neuron activity was observed for the neuron illustrated in Figure 8. This neuron, like that 

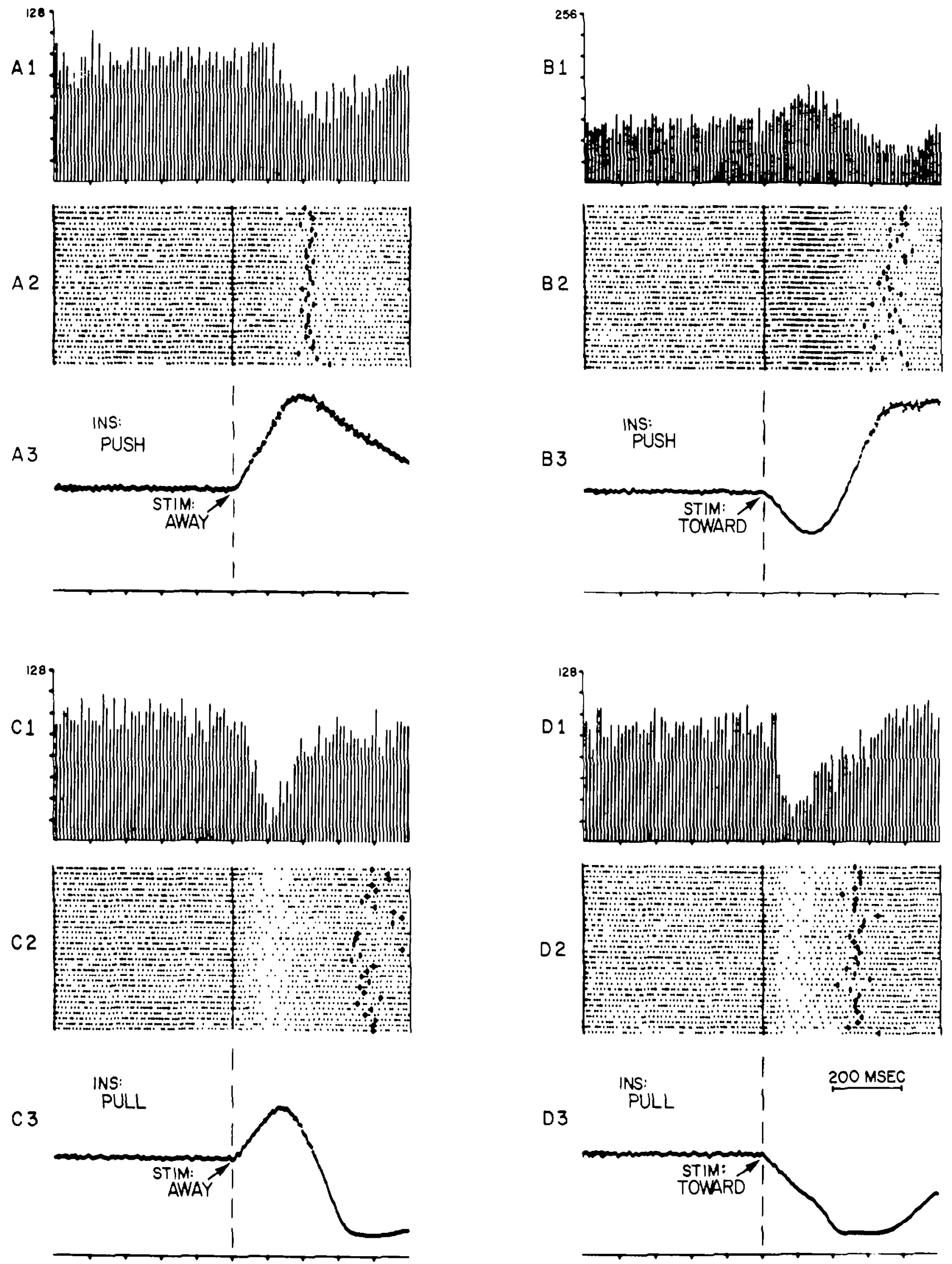

Figure 6. Responses of a dentate neuron to imposed displacements: Modification due to motor preparation. The conventions in this figure are identical to those in Figure 5 . The maximum of the scale in B1 (256) differs from that of the other averages (128). Note that imposed displacements of the handle (away from or toward the monkey) evoked a short latency decrease in activity only when the animal was prepared to pull. 

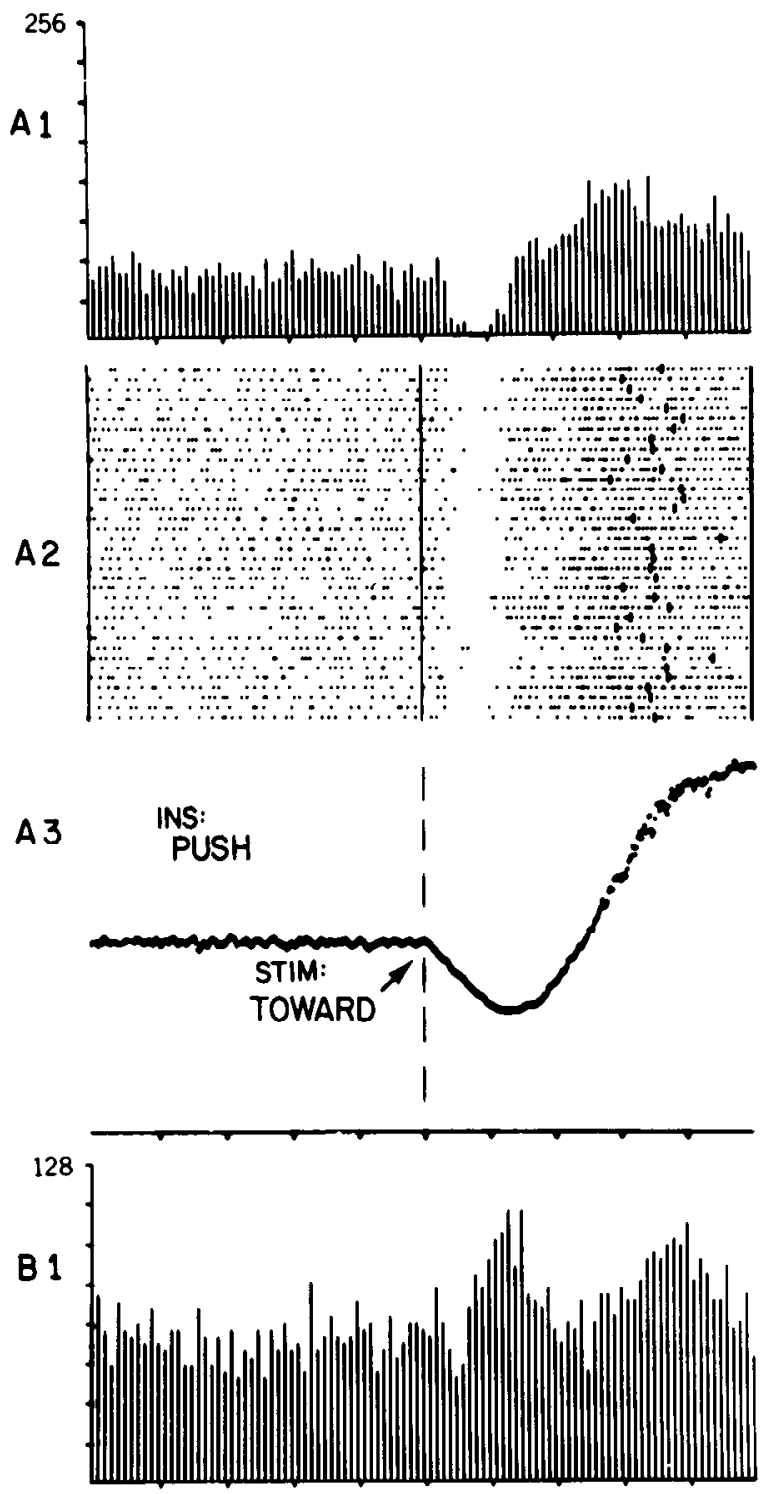

2
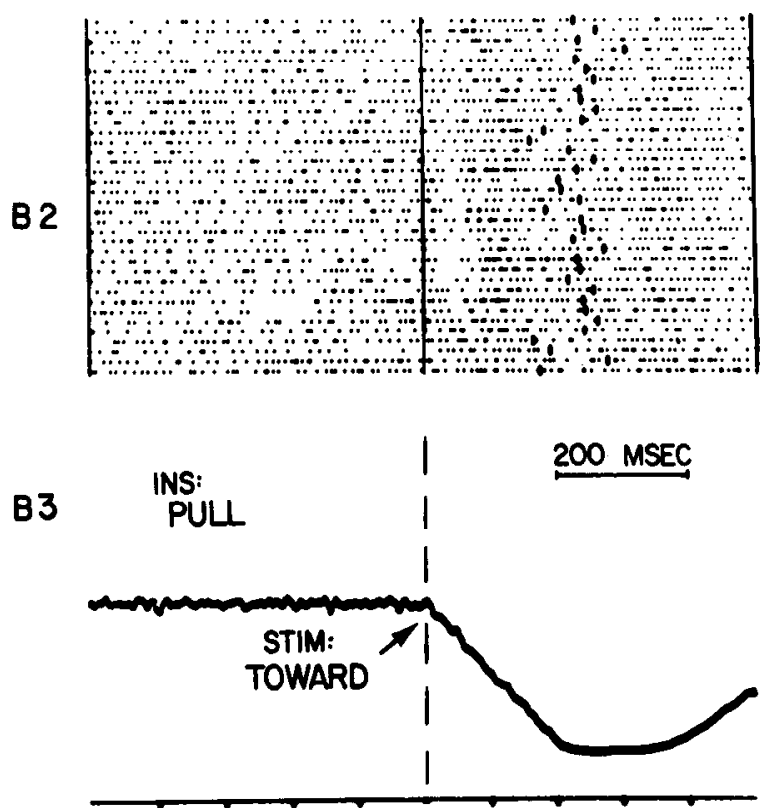

Figure 7. Responses of a second type of dentate neuron to imposed displacements: Evoked decrease depends on motor preparation and the direction of the imposed displacement. In illustrated in Figure 7, had a short latency response only with one combination of instruction and imposed displacement. In this case there was a short latency increase in activity only when the animal was instructed to prepare to push and the imposed displacement moved the handle away from the monkey (Fig. 8A). Since the direction of the imposed displacement was the same in Figure $8, A$ and $B$, the lack of a response during the analysis period in Figure $8 B$ can be due only to the different prior instructions.

Figure 9 illustrates the responses of a dentate neuron with a complicated pattern of activity in the analysis period. The only condition in which there was a short latency increase in activity was when the animal was instructed to prepare to push and the imposed displacement moved the handle away from the monkey (Fig. 9A). Short latency decreases in activity were evoked in the remaining three conditions. The most dramatic decrease occurred when the animal was instructed to prepare to pull and the imposed displacements moved the handle toward the monkey (Fig. $9 D$ ). Thus, the largest changes in activity for this neuron in the analysis period occurred when the imposed displacements moved the handle in the same direction as the animal was instructed to move (Fig. 9A (increase) and Fig. 9D (decrease)). Although the precise factors which determined this neuron's pattern of response are unclear, its response does illustrate that complicated interactions between imposed displacements and prior instructions can occur.

\section{Discussion}

The primary goal of this study was to examine an interaction between peripheral afferent input and central command signals at the level of cerebellar output. This was accomplished by analyzing how the motor preparation or "set" of an animal to move in a particular direction influenced the response evoked in cerebellar output neurons by somatosensory afferent inputs. It was possible to evoke short latency responses from both dentate and interpositus neurons with a kinesthetic input, i.e., a torque motor-imposed displacement of the animal's limb. The major new observation of the present study is that motor preparation markedly influenced the response of many dentate neurons to kinesthetic inputs and had little or no influence on the response of most interpositus neurons.

The influence of motor preparation was seen in more than two-thirds of those dentate neurons that displayed a neural response to kinesthetic inputs. For most of the neurons whose activity was influenced by motor preparation, their neural response was contingent on two factors: (1) the prior instruction given to the animal and (2) the direction of the imposed limb displacement. Thus, the response of these neurons reflected the result of a complex interaction between motor preparation and afferent input.

both $A$ and $B$, the imposed displacement moved the handle toward the monkey. Imposed displacements away from the monkey (not illustrated) failed to evoke a short latency response. The maximum of the scale in $B 1(128)$ differs from that in $A 1(256)$. 


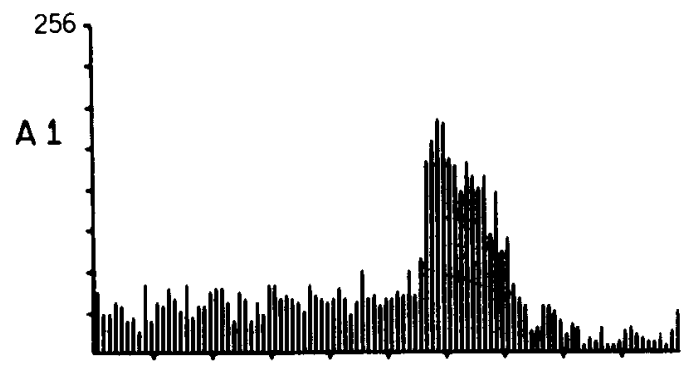

A2
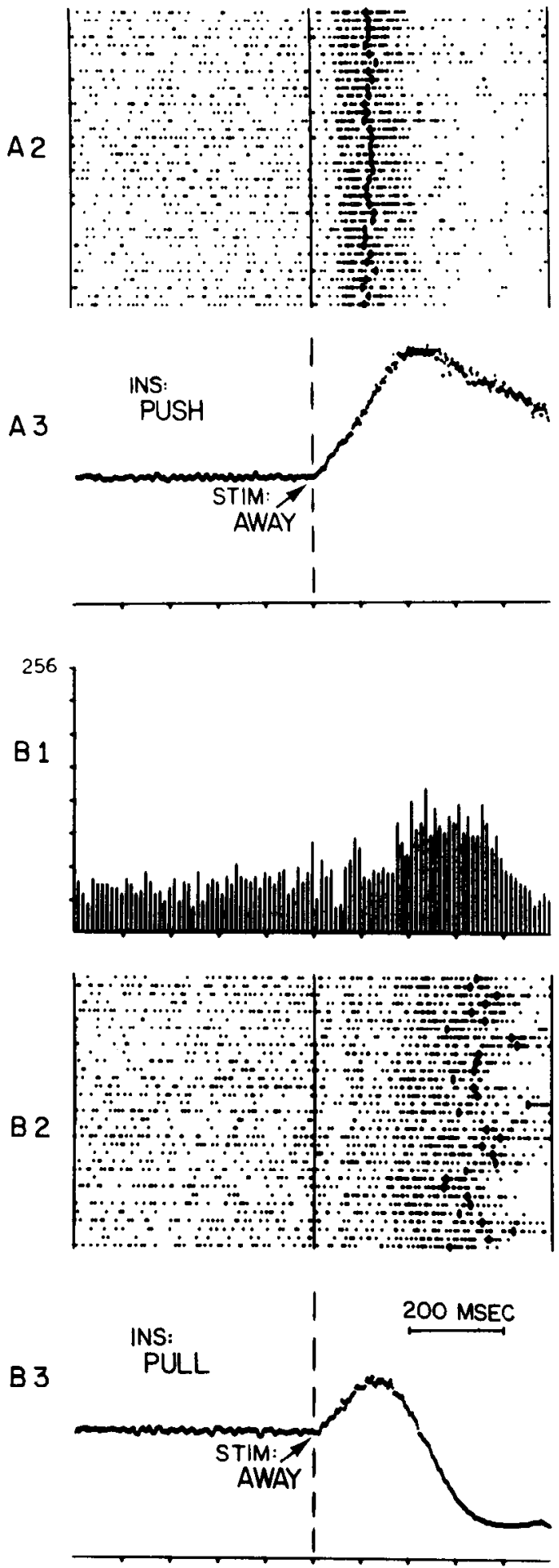

Figure 8. Responses of a second type of dentate neuron to imposed displacements: Evoked increase depends on motor
In an additional set of dentate neurons (13\% of those influenced by motor preparation), the characteristics of their short latency response were determined solely by the nature of the prior instruction. For these neurons, the imposed displacement served as a trigger for possible changes in activity and appeared to have little influence on determining the pattern of response. This second group of neurons is especially interesting because their response patterns are consistent with those of a motor command signal. Namely, although triggered by afferent input, their activity: (1) is independent of the parameters of this input, (2) is related to the motor task, and (3) precedes the motor task. These neurons could be important components of the neural circuitry which generates motor responses that are not tightly coupled, in a reflexive manner, to the afferent inputs which triggered them.

Unlike dentate responses, the short latency response of interpositus neurons to kinesthetic inputs was largely unaffected by differing motor sets. Instead, for more than $60 \%$ of the interpositus neurons that responded at short latency to kinesthetic inputs, the response was strongly influenced by the direction of the imposed limb displacement. These observations suggest that the short latency responses of many interpositus neurons to kinesthetic inputs may provide a reliable signal of the activity in peripheral afferents related to limb movement. Previous studies of the deep cerebellar nuclei during movement have observed similar differences between dentate and interpositus neuron activity (e.g., Thach, 1970, 1975, 1978).

The striking differences in the response patterns of dentate and interpositus neurons suggest that each may differentially contribute to motor output and, in particular, to the activity of motor cortex neurons during the task. The timing and activity patterns of dentate neurons in the present experiment are consistent with their contributing to the control and/or generation of those components of task-related muscle activity which, although triggered by a limb displacement, are dependent on the motor preparation of the animal (e.g., the "M3" and "voluntary" responses described by Tatton et al., 1975; see also Evarts and Granit, 1976; Marsden et al., 1978). This suggestion is consistent with the reports that : (1) experimental cooling of cerebellar nuclei in trained monkeys delays "predictive" muscle responses which are triggered by limb displacements (Vilis and Hore, 1977, 80), and (2) short latency "voluntary or triggered responses" evoked by limb displacements are reduced or absent in the muscles of humans showing symptoms of cerebellar damage (Marsden et al., 1977; MacKay and Murphy, 1979). Although there is considerably less supporting evidence, similar arguments can be made for a contribution of interpositus neurons to the control and/or generation of muscle activity which appears to be more reflexive in nature (e.g., the "M2" response of Tatton et al., 1975). However, any further discussion of this topic

preparation and the direction of the imposed displacement. In both $A$ and $B$, the imposed displacement moved the handle away from the monkey. Imposed displacements toward the monkey (not illustrated) failed to evoke a short latency response. 

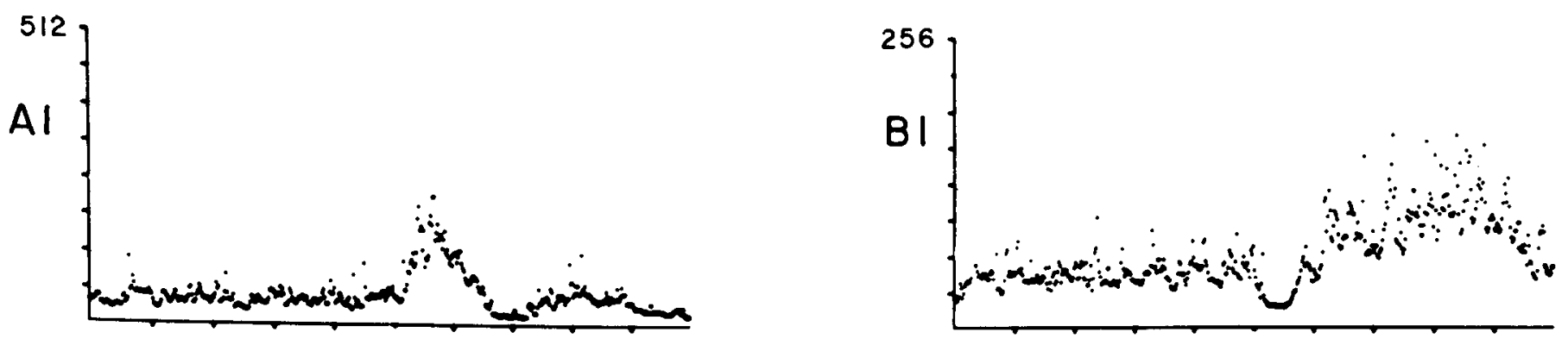

A2

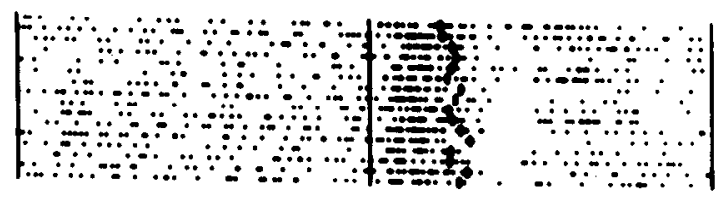

82
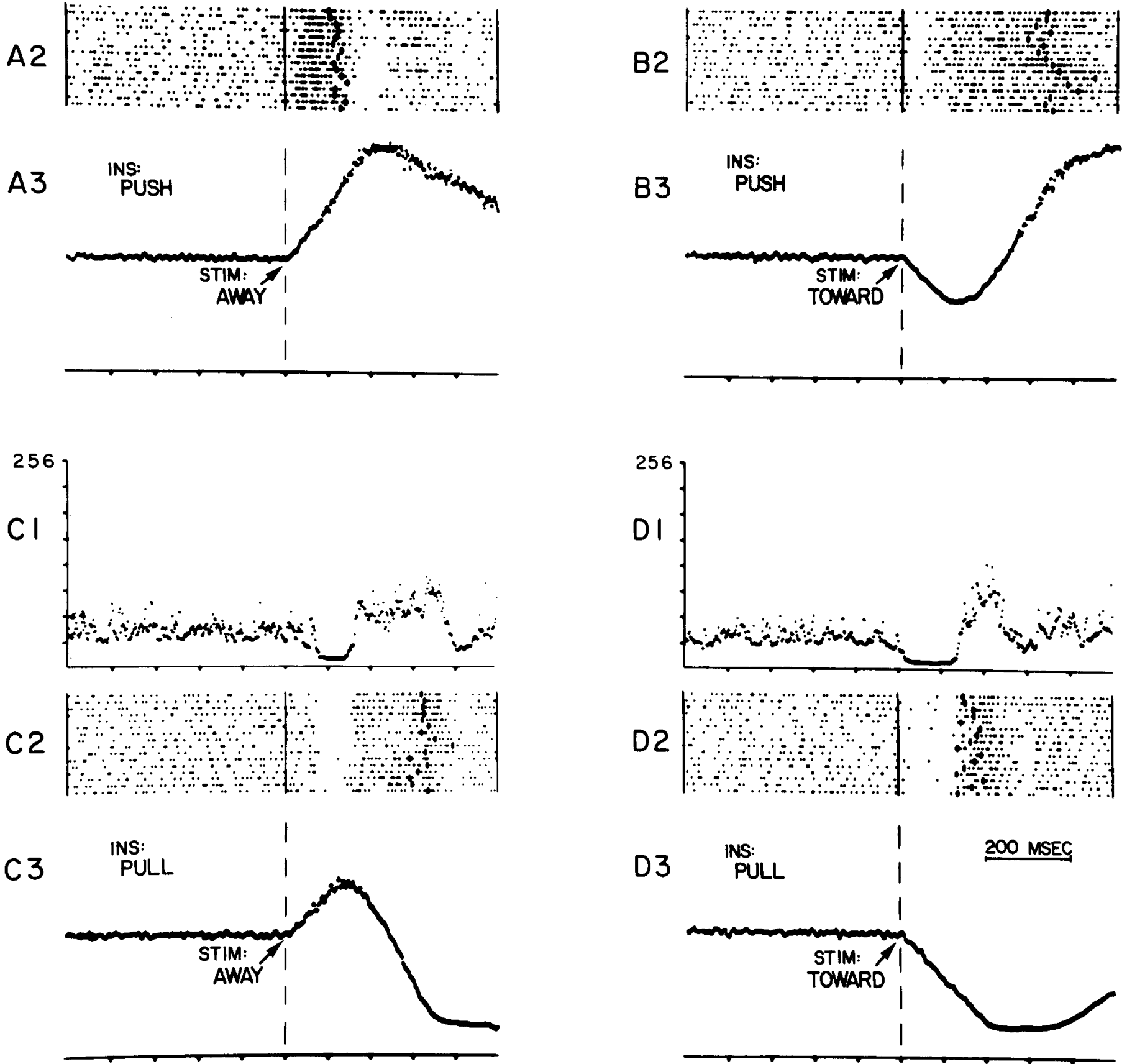

Figure 9. Responses of a dentate neuron to imposed displacements: Evoked responses depend on a complex interaction between the direction of the imposed displacement and motor preparation. The conventions in this figure are identical to those in Figure 5. The maximum of the scale in $A 1$ (512) differs from that of the other averages (256). Note that a short latency increase in this neuron's activity occurred only when the handle was moved away from the monkey and the animal was prepared to push. The largest decrease in activity occurred when the handle was moved toward the monkey and the animal was prepared to pull. 
is complicated by the present uncertainties concerning the relative contributions of "long-loop" and spinal mechanisms to the generation of muscle responses triggered by limb displacements (see, for example, Ghez and Shinoda, 1978, and Miller and Brooks, 1981, for references). Therefore, the remainder of this discussion will focus on the potential contribution of cerebellar activity to the generation of motor cortex responses.

As noted in the introduction, Evarts and Tanji (1976) have recorded the activity of neurons in the motor cortex of animals trained to perform the same task as that employed in this experiment. They observed that imposed displacements could evoke two types of responses from pyramidal tract neurons (PTNs). The earliest response, termed a "reflex PTN discharge," occurred at latencies as short as $20 \mathrm{msec}$. This response depended on the direction of the imposed displacement and was not influenced by the prior motor preparation of the animal. A longer latency response, termed an "intended PTN discharge," depended on the direction of the movement which the animal performed following an imposed displacement and, thus, depended on the nature of the prior instruction. Unlike the "reflex" response, the "intended discharge" was not influenced by the direction of the imposed displacement.

There are obvious similarities between the "reflex" response in motor cortex and the response of interpositus neurons, and between the "intended" response in the motor cortex and the response of dentate neurons. For example, the short latency response of interpositus neurons and the PTN "reflex" response both were dependent on the direction of the imposed displacement and not influenced by the motor set of the animal. On the other hand, the response of dentate neurons and the PTN "intended" response both were influenced by the animal's motor set. Thus, "reflex" and "intended" responses which are represented separately in the dentate and interpositus appear to be combined at the level of the motor cortex.

Given the projections of the deep cerebellar nuclei to thalamic regions which project to the motor cortex (e.g., Kievil and Kuypers, 1972; Percheron, 1977; Stanton, 1980; Kalil, 1981), it seems reasonable to suggest that outputs from dentate and interpositus could participate in the generation of the two phases of motor cortex response. The timing of cerebellar and motor cortex responses supports this suggestion. The onset of the earliest response evoked from interpositus neurons by imposed displacements $(18 \mathrm{msec})$ preceded the earliest "reflex" response of PTNs $(20 \mathrm{msec})$, and the onset of the earliest response evoked from dentate neurons (27 $\mathrm{msec}$ ) preceded the earliest "intended" response (44 $\mathrm{msec}$ ). However, there are many reasons to interpret these small differences in timing with caution. For example, the cortical and cerebellar recordings were done in different animals, and there was considerable overlap in the timing of cerebellar and motor cortex responses.

The results of cerebellar cooling in primates and cats, however, further support the suggestion that cerebellar responses to limb displacements (especially those of dentate) could contribute to the generation of motor cortex responses (primate studies: Meyer-Iohmann et al, 1975; Vilis et al., 1976; cat study: Murphy et al., 1975). In the primate experiments, monkeys were trained to perform self-paced movements of a handle between two target zones using flexion and extension of the elbow. Imposed displacements (brief rectangular force pulses) were applied to the handle near the beginning of randomly selected movements (see Conrad ct al., 1974, 1975, for details). The imposed displacements evoked two types of responses from motor cortex neurons termed "early" and "late" responses. "Early" responses were positively correlated with the duration, intensity, and, for two-thirds of the neurons, direction of the imposed displacement. "Late" responses correlated with later, variable "corrective movements" which the monkey performed to compensate for the imposed displacements. Thus, "early" and "late" responses were comparable to the PTN "reflex" and "intended" responses of Evarts and Tanji (1976).

Reversible cooling of the deep cerebellar nuclei during performance of the task differentially affected the two cortical responses. Dentate cooling (Meyer-Lohmann et al., 1975) markedly reduced and/or delayed only the "late" response evoked from motor cortex neurons by limb displacement. Smaller effects were observed on the "early" response only following interpositus cooling (Vilis et al., 1976). Interpositus cooling also affected the "late" response. However, cooling through the probe implanted in interpositus also affected dentate neurons (Vilis et al., 1976), and the authors could not rule out the possibility that the modification of the "late" response arose entirely from dentate cooling as in their earlier experiment (Meyer-Lohmann et al., 1975). Thus, the major effect of cerebellar cooling was on the "late" responses and this effect probably arose from cooling the dentate nucleus.

If cerebellar output contributes to the generation of "late" or "intended" responses in the motor cortex, then this influence is likely to be mediated via cerebellothalamocortical pathways traversing the ventrolateral nucleus of the thalamus (VL). Recordings in awake monkeys show that VL neurons, while well related to active limb movements, were largely uninfluenced by gentle passive manipulation of the animal's limb (Joffroy and Lamarre, 1974; Strick, 1976b; Macpherson et al., 1980). Responses to passive manipulation would be expected if these neurons were to contribute to the "reflex" response in the motor cortex. The activity of some VL neurons, however, was modified between 30 and $50 \mathrm{msec}$ after the imposition of small load perturbations applied to the handle which the animal was holding (Strick, 1976b). These responses appeared to relate more to the animal's corrective movements which followed the perturbation than to the perturbation itself. Responses of this type are what would be expected if these neurons were to contribute to PTN “intended" responses. Furthermore, the latency of the VL responses suggests that, although they were too late to contribute to "reflex" responses in the motor cortex, they were early enough to contribute to "intended" responses. Thus, the results of recordings from thalamic neurons are consistent with VL mediating 
the influence of the dentate nucleus on PTN "intended" responses, but provide little evidence for a VL contribution to PTN "reflex" responses.

Although the preceding discussion has focused on the issue of dentate and interpositus contributions to motor cortex responses, clearly other potential routes exist for generating PTN "reflex" and "intended" responses. A complete discussion of the alternative pathways is beyond the scope of this paper; thorough reviews of these issues have recently been presented by Asanuma (1981) and Evarts (1981).

The results of the present study give little insight into the mechanisms that might be utilized during motor preparation to control the short latency response of dentate neurons to kinesthetic inputs. One potential mechanism would be to change the tonic excitability of dentate neurons in relation to the preparation to move in a particular direction. Tonic changes in neural activity prior to movement have been observed in various cortical and deep cerebellar structures (e.g., Kornhuber and Deecke, 1965; Kubota and Niki, 1971; Fuster, 1973; Kutas and Donchin, 1974; Tanji and Evarts, 1976; Thach, 1978; Tanji et al., 1980). However, changes in tonic activity can be ruled out because no neural responses specifically related to the instruction were observed in the present study. It seems more likely that neurons relaying phasic inputs to the dentate nucleus were modified during the instruction period, although other possibilities certainly exist.

Whatever the mechanisms responsible for the modifications in dentate responses, part of the process of developing a motor set to move in a particular direction involves "preprogramming" the response of dentate neurons to kinesthetic inputs. The results of this process is that dentate neuron activity behaves like a motor command signal which is triggered at short latency by a peripheral event. The special characteristic of this signal is that it is dependent on the intent of the animal and not on the qualities of the triggering event. Since the intent of an animal constantly varies, dentate neurons may provide an output which can rapidly adapt motor responses to meet these changing internal conditions.

\section{References}

Asanuma, H. (1981) The pyramidal tract. In Handbook of Physiology: The Nervous System, Vol. II: Motor Control, V. B. Brooks, ed., pp. 703 733, Williams \& Wilkins, Baltimore. Conrad, B., K. Matsunami, J. Meyer-Lohmann, M. Wiesendanger, and V. B. Brooks (1974) Cortical load compensation during voluntary elbow movements. Brain Res. 71: 507-514.

Conrad, B., J. Meyer-Lohmann, K. Matsunami, and V. B. Brooks (1975) Precentral unit activity following torque pulse injections into elbow movements. Brain Res. 94: 219-236.

Crago, P. E., J. C. Houk, and Z. Hazan (1976) Regulatory actions of the human stretch reflex. J. Neurophysiol. 39: 925935.

Creed, R. S., D. Denny-Brown, J. C. Eccles, E. G. T. Liddell, and C. S. Sherrington (1932) Reflex Activity of the Spinal Cord, Oxford University Press, London.

Evarts, E. V. (1968) A technique for recording activity of subcortical neurons in moving animals. Electroencephalogr. Clin. Neurophysiol. 24: 83-86.
Evarts, E. V. (1981) Role of the motor cortex in voluntary movements in primates. In Handbook of Physiology: The Nervous System, Vol. II: Motor Control, V. B. Brooks, ed., pp. 1083-1120, Williams \& Wilkins, Baltimore.

Evarts, E. V., and R. Granit (1976) Relations of reflexes and intended movements. Prog. Brain Res. 44: 1-14.

Evarts, E. V., and J. Tanji (1974) Gating of motor cortex reflexes by prior instruction. Brain Res. 71: 479-494.

Evarts, E. V., and J. Tanji (1976) Reflex and intended responses in motor cortex pyramidal tract neurons of monkey. J. Neurophysiol. 39: 1069-1080.

Evarts, E. V., and W. J. Vaughn (1978) Intended arm movements in response to externally produced arm displacements in man. Prog. Clin. Neurophysiol. 4: 178-192.

Evarts, E. V., E. Bizzi, R. E. Burke, M. DeLong, and W. T. Thach (1971) Central control of movement. Neurosci. Res. Program Bull. 9: 129-135.

Fetz, E. E., D. V. Finocchio, M. A. Baker, and M. J. Soso (1980) Sensory and motor responses of precentral cortex cells during comparable passive and active joint movements. J. Neurophysiol. 43: 1070-1089.

Fuster, J. M. (1973) Unit activity in prefrontal cortex during delayed response performance: Neuronal correlates of transient memory. J. Neurophysiol. 36: 61-78.

Ghez, C., and Y. Shinoda (1978) Spinal mechanisms of the functional stretch reflex. Exp. Brain Res. 32: 55-68.

Hammond, P. H. (1956) The influence of prior instruction to the subject on an apparently involuntary neuro-muscular response. J. Physiol. (Lond.) 132: 17-18P.

Hammond, P. H. (1960) An experimental study of servo action in human muscular control. In Proceedings of the Third International Conference in Medical Electronic, pp. 190-199, Institute of Electronic Engineering, London.

Houk, J. C. (1978) Participation of reflex mechanisms and reaction time processes in the compensatory adjustments to mechanical disturbances. Prog. Clin. Neurophysiol. 4: 193215.

Joffroy, A. J., and Y. Lamarre (1974) Single cell activity in the ventral lateral thalamus of the unanesthetized monkey. Exp. Neurol. 42: 1-16.

Kalil, K. (1981) Projections of the cerebellar and dorsal column nuclei upon the thalamus of the rhesus monkey. J. Comp. Neurol. 195: 25-50.

Kievit, J., and H. G. J. M. Kuypers (1972) Fastigial cerebellar projections to the ventrolateral nucleus of the thalamus and the organization of the descending pathways. In Corticothalamic and Sensorimotor Activities, T. Frigyesi, E. Rinvik, and M. D. Yahr, eds., pp. 91-114, Raven Press, New York.

Kornhuber, H. H., and L. Deecke (1965) Hirnpotentialanderungen bei Willkurbewegungen und passiven Bewegungen des Menschen: Bereitschaf potential und reafferente Potentiale. Arch. Gesante Physiol. 284: 1-17.

Kubota, K., and H. Niki (1971) Prefrontal cortical unit activity and delayed alternation performance in monkeys. J. Neurophysiol. 34: 337-347.

Kutas, M., and E. Donchin (1974) Studies of squeezing: Handedness, responding hand, response force, and asymmetry of readiness potential. Science 186: $545-548$.

Lemon, R. N., and R. Porter (1976) Afferent input to movement related precentral neurones in conscious monkeys. Proc. R. Soc. Lond. Biol. 194: 313-339.

MacKay, W. A., and J. T. Murphy (1979) Cerebellar influence on proprioceptive control loops. In Cerebro-Cerebellar Interactions, J. Massion and K. Sasaki, eds., pp. 141-162, ElsevierNorth Holland Publishing Co., Amsterdam.

Macpherson, J. M., D. D. Rasmusson, and J. T. Murphy (1980) Activities of neurons in the "motor" thalamus during control 
of limb movement in the primate. J. Neurophysiol. 44: 1128.

Marsden, C. D., P. A. Merton, H. B. Morton, M. Hallet, J. Adam, and D. N. Rushton (1977) Disorders of movement in cerebellar disease in man. In Physiological Aspects of Clinical Neurology, F. C. Rose, ed., pp. 179-199, Rlackwell, Oxford.

Marsden, C. D., P. A. Merton, H. B. Morton, J. Adam, and M. Hallett (1978) Automatic and voluntary responses to muscle stretch in man. Prog. Clin. Neurophysiol. 4: 167-177.

Meyer-Lohmann, J., B. Conrad, K. Matsunami, and V. B. Brooks (1975) Effects of dentate cooling on precentral unit activity following torque pulse injections into elbow movements. Brain Res. 94: 237-251.

Miller, A. D., and V. B. Brooks (1981) Late muscular responses to arm perturbations persist during supraspinal dysfunctions in monkeys. Exp. Brain Res. 41: 146-158.

Murphy, J. T., Y. C. Wong, and H. C. Kwan (1975) Afferentefferent linkages in motor cortex for single forelimb muscles. J. Neurophysiol. 38: 990-1014.

Percheron, G. (1977) The thalamic territory of cerebellar afferents and the lateral region of the thalamus of the macaque in stereotaxic ventricular coordinates. J Hirnforsch. 18: 375400.

Rosen, I., and H. Asanuma (1972) Peripheral afferent inputs to the forelimb area of the monkey motor cortex: Inputoutput relations. Exp. Brain Res. 11: 257-273.

Stanton, G. B. (1980) Topographical organization of ascending cerebellar projections from dentate and interposed nuclei in Macaca mulatta: An anterograde degeneration study, J. Comp. Neurol. 190: 699-731.

Strick, P. L. (1976a) Anatomical analysis of ventrolateral thalamic input to primate motor cortex. J. Neurophysiol. 39: 1020-1031.

Strick, P. L. (1976b) Activity of ventrolateral thalamic neurons during arm movement. J. Neurophysiol. 39: 1032-1044.

Strick, P. L. (1976c) Cercbellar neuron response to imposed limb displacement: Dependence of short latency dentate activity on intended movement. Soc. Neurosci. Abstr. 2: 876.

Strick, P. L. (1978) Cerebellar involvement in 'volitional' muscle responses to load changes. Prog. Clin. Neurophysiol. 4:
85-93.

Strick, P. L., and J. B. Preston (1982) Two representations of the hand in area 4 of a primate. II. Somatosensory input organization. J. Neurophysiol. 48: 150-159.

Tanji, J., and E. V. Evarts (1976) Anticipatory activity of motor cortex neurons in relation to direction of an intended movement. J. Neurophysiol. 39: 1062-1068.

Tanji, J., K. Taniguchi, and T. Saga (1980) Supplementary motor area: Neuronal response to motor instructions. J. Neurophysiol. 43: 60-68.

Tatton, W. G., S. D. Forner, G. L. Gerstein, W. W. Chambers, and C. N. Liu (1975) The effect of postcentral cortical lesions on motor responses to sudden upper limb displacements in monkeys. Brain Res. 96: 108-113.

Thach, W. T. (1970) Discharge of cerebellar neurons related to two maintained postures and two prompt movements. I. Nuclear cell output. J. Neurophysiol. 33: 527-536.

Thach, W. T. (1975) Timing of activity in cerebellar dentate nucleus and cerebral motor cortex during prompt volitional movement. Brain Res. 88: 233-241.

Thach, W. T. (1978) Correlation of neural discharge with pattern and force of muscular activity, joint position, and direction of intended next movement in motor cortex and cerebellum. J. Neurophysiol. 41: 654-676.

Villis, T., and J. Hore (1977) Effects of changes in mechanical state on cerebellar intention tremor. J. Neurophysiol. 40 . 1214-1224.

Villis, T., and J. Hore (1980) Central neural mechanisms contributing to cerebellar tremor produced by limb perturbations. J. Neurophysiol. 43: 279-291.

Villis, T., J. Hore, J. Meyer-Lohmann, and V. B. Brooks (1976) Dual nature of the precentral responses to limb perturbations revealed by cerebellar cooling. Brain Res. 117: 336-340.

Wolbarsht, M. L., E. F. MacNichol, and H. G. Wagner (1960) Glass insulated platinum microelectrodes. Science 132: 1309 1310.

Wong, Y. C., H. C. Kwan, W. A. MacKay, and J. T. Murphy (1978) Spatial organization of precentral cortex in awake primates. I. Somatosensory inputs. J. Neurophysiol. 41: 1107-1119. 